\title{
IMPORTANCIA DE LAS MICORRIZAS ARBUSCULARES (MA) PARA UN USO SOSTENIBLE DEL SUELO EN LA AMAZONIA COLOMBIANA
}

\author{
Lina Paola Garzón ${ }^{1}$
}

Recibido el 4 de octubre de 2014, aprobado el 19 de marzo de 2015 y actualizado el 09 de noviembre de 2015

DOI: 10.17151/luaz.2016.42.14

\section{RESUMEN}

La Amazonia colombiana es una fuente de diversidad que se ha visto afectada por cultivos comerciales insostenibles que afectan la actividad de la microbiota del suelo $y$, por ende, el crecimiento de las especies vegetales. La limitación de fósforo y nutrientes en el suelo debe ser compensada por el sistema radicular de las plantas y potenciada por la presencia de las micorrizas arbusculares que aumentan su nivel de movilización.

En este sentido, el conocimiento sobre las características fisicoquímicas del suelo y su relación con la presencia de micorrizas, es fundamental para generar procesos agrícolas más productivos y con menor impacto ambiental. Este artículo hace un análisis de publicaciones científicas entre 1995 y 2013 sobre la importancia de las micorrizas para un uso sostenible del suelo en la Amazonia colombiana, dando a conocer su funcionalidad en la estabilidad edáfica y su contribución para una agricultura comercial sostenible.

Las micorrizas se encuentran ampliamente distribuidas en el suelo amazónico y su relación sinérgica con otros microorganismos favorece la absorción de nutrientes en las plantas. Asimismo, pueden ser de gran potencial en la agricultura sostenible como fertilizantes orgánicos; aunque, se reconoce que falta investigar más acerca de las comunidades nativas de hongos formadores de micorrizas, el potencial micorrízico del suelo y sus niveles nutricionales.

\section{PALABRAS CLAVE}

Agrosistema, Amazonas, micología, recursos de suelos, sostenibilidad.

\section{IMPORTANCE OF ARBUSCULAR MYCORRHIZAE FOR SUSTAINABLE LAND USE IN THE COLOMBIAN AMAZON RAINFOREST}

\section{ABSTRACT}

The Colombian Amazon is a source of diversity that has been affected by unsustainable commercial crops that have a negative effect on the activity of the soil microbiota and therefore, on the growth of plants. Limited phosphorus and nutrients in the soil must be compensated by the plant root system and enhanced by arbuscular mycorrhizae to increase their level of mobilization. In this sense, the knowledge of the physicochemical characteristics of the soil and its relationship with the presence of mycorrhizae is essential to generate more productive agricultural processes with less environmental impact. This article analyzes scientific publications between 1995 and 2013 which deal with the importance of mycorrhizae for a sustainable land use in the Colombian Amazon, thus 
revealing its functionality on edaphic stability and its contribution to sustainable commercial agriculture. Mycorrhizae are widely distributed in the Amazonian soil and its synergic relationship with other microorganisms favors the absorption of nutrients in plants. Additionally, they can be a great potential in sustainable agriculture as organic fertilizers though, more research is needed about the native communities of mycorrhizal fungi, mycorrhizal soil potential and its nutritional levels.

KEY WORDS: Agrosystem, Amazon, mycology, soil resources, sustainability.

\section{INTRODUCCIÓN}

El Amazonas actualmente es considerado como una de las mayores reservas de biodiversidad en el mundo (Jesus et al., 2009), especialmente de especies vegetales que dependen de una gran variedad de microorganismos presentes en el suelo. Sin embargo, la presión de las actividades antropogénicas como los cultivos comerciales extensivos e insostenibles, ha llevado a una pérdida de la microbiota afectando la disposición de materia orgánica esencial para el crecimiento vegetal y, en consecuencia, ha ocasionado una alteración del equilibrio ecosistémico (Fracetto et al., 2013).

De igual forma, el uso de pesticidas y fertilizantes inorgánicos en agroecosistemas amazónicos ha generado problemas ambientales por la contaminación de cuerpos de agua y su posterior eutroficación (Cuenca et al., 2007). Esto ha llevado a un creciente interés por la implementación de una fertilización ecológica, basada en el uso de microorganismos beneficiosos como las micorrizas (Barrer, 2009). Sin embargo, hace falta investigar más acerca de las comunidades nativas de hongos formadores de micorrizas, el potencial micorrízico del suelo y sus niveles nutricionales, con el fin de potenciar su uso sostenible en la agricultura amazónica colombiana (Cuenca et al., 2007).

La microbiota realiza una serie de funciones clave para mantener la productividad, diversidad y estructura de las comunidades vegetales en el planeta (Van der Heijden et al., 2008), puesto que actúa como una proveedora de nutrientes que son absorbidos por las plantas. Entre los organismos que habitan en el suelo se pueden destacar por su función ecológica los hongos formadores de micorrizas, los cuales pueden tener una alta incidencia en la estabilidad de ecosistemas donde las condiciones edáficas son extremas (Martínez y Pugnaire, 2009).

En este sentido, la micorriza es una simbiosis mutualista que tiene como función aumentar la superficie de absorción de la raíz, por medio de un sistema de hifas extrarradicales (Castro, 2009). La planta puede absorber y asimilar más agua, minerales (nitrógeno y fósforo) e iones poco móviles (ácido fosfórico, amoniaco, zinc, cobre), favoreciéndose su balance hídrico y nutrición (Barrer, 2009).

En la Amazonia colombiana los suelos se caracterizan por ser pobres tanto en materia orgánica como en nutrientes. Por esta condición, las micorrizas han llegado a considerarse casi como una relación obligada para el crecimiento de poblaciones vegetales, tanto en condiciones naturales como en agrosistemas (León, 2006). Esto ha llevado a 
contemplarlas como una herramienta de manejo sostenible del suelo pues contribuyen con: aumento de productividad de los cultivos, regeneración de comunidades vegetales degradadas y mantenimiento del equilibrio del ecosistema (Guerra, 2008).

Múltiples estudios alrededor del mundo han enfocado las micorrizas arbusculares en el área de la agricultura sostenible como mecanismo para mantener cultivos comerciales eficientes y sostenibles (Cuenca et al., 2007; Guerra, 2008; Phosri et al., 2010; Fitter, Helgason y Hodge, 2011; Pellegrino et al., 2011; Andrews, Cripps y Edwards, 2012).

Este artículo tiene por objetivo hacer un análisis de las publicaciones científicas entre los años 1995 y 2013 sobre la importancia de las micorrizas para un uso sostenible del suelo en la Amazonia colombiana. Se pretende, adicionalmente, dar a conocer la funcionalidad de los hongos micorrízicos en la estabilidad edáfica y su contribución para una agricultura comercial sostenible.

\section{Asociaciones microbianas y su relación con los hongos formadores de micorrizas (HFMA)}

Los suelos de la Amazonia son pobres tanto en materia orgánica como en nutrientes, ya que la capa orgánica es delgada y poco descompuesta por lo que se presenta poca humificación del suelo (Cardona, 2000). Adicionalmente, los nutrientes se encuentran en la capa de la hojarasca y en el dentritus, en donde las plantas los obtienen a partir de las raíces alimentadoras y los hongos micorrízicos (Peña-Venegas et al., 2006).

El fósforo es considerado el elemento limitante para el desarrollo de las plantas, debido a la madurez de los suelos amazónicos, en los cuales se encuentran grandes cantidades de óxido de hierro y aluminio que promueven la formación de los fosfatos. De hecho, de las formas minerales de este elemento el $60-70 \%$ se encuentra en forma de fosfatos de hierro, los cuales son solubilizados por los microorganismos para liberar fósforo inorgánico y otras formas solubles que se encuentran disponibles para las plantas (Useche, Valencia y Pérez, 2004).

Asimismo, la materia orgánica es la principal fuente de fósforo en los suelos de la Amazonia, por lo cual el fósforo orgánico constituye el 30$50 \%$ del total del fósforo presente en el suelo. De igual forma, la biomasa bajo el suelo tiende a constituir el 32\% del nitrógeno total en los bosques tropicales, por lo cual su valor es significativo en estos ecosistemas que dependen estríctamente de reciclaje de materia orgánica en descompsición (Peña-Venegas y Cardona, 2010).

Debido a estas condiciones edáficas particulares, la existencia e interacción de las comunidades microbiológicas del suelo amazónico son complejas y críticas para mantener la funcionalidad del bioma (Fracetto et al., 2013), puesto que tienen incidencia directa en los ciclos biogeoquímicos especialmente en el ciclo del nitrógeno y en la absorción del fósforo (Rodrigues et al., 2012); así como en la producción de sustancias útiles y remoción de toxinas, lo que permite mantener la estabilidad de la vegetación nativa (Toro, 2004; Dilantha y Ru, 2012).

Esta actividad microbiológica edáfica puede ser llevada a cabo por asociaciones mutualistas entre hongos micorrízicos con otras especies de bacterias y hongos, por lo que de forma sinérgica pueden contribuir en el 
control biológico de fitopatógenos y en la estimulación del crecimiento vegetal (Cano, 2011; King, 2011). Por ejemplo, Bharadwaja et al. (2008) encontraron que la colonización de las raíces con HFMA puede incrementarse en presencia de bacterias como Pseudomonas, Stenotrophomonas y Arthrobacter, lo que permite una inhibición del crecimiento de fitopatógenos, tales como Erwinia carotovora, Phytophthora infestans y Verticillium dahliae.

Asimismo, en los ecosistemas tropicales la absorción del fósforo ocurre pobremente sobre la superficie del suelo, por lo cual las plantas y los microrganismos son los encargados de inmovilizar este elemento. Por ejemplo, los hongos saprófitos se encargan de la fase no soluble del fósforo en la materia orgánica, compuesta por ácidos nucleicos, fosfolípidos y fosfoproteínas; más adelante, esta labor es continuada por los hongos de crecimiento lento (Peña-Venegas et al., 2006).

Se estima que entre el 10 y 50\% de las bacterias presentes en la rizósfera tienen la capacidad de solubilizar fosfatos de calcio, dentro de las cuales se encuentran las especies de Pseudomonas, Mycobacterium, Micrococcus, Arthrobacter y Flavobacterium (Peña-Venegas y Cardona, 2010). Algunos estudios en suelos del sur de la Amazonia colombiana (Cabrera, 2000; Useche et al., 2004) determinaron que el 5,5\% de las bacterias $y$ el $3,2 \%$ de los hongos (especialmente de las especies Penicillium, Aspergillus, Scytalidium y Paecilomyces) tienen capacidad solubilizadora de fosfatos de calcio en Ultisoles y Oxisoles.

Otros microorganismos son capaces de solubilizar compuestos insolubles de fósforo con hierro (estrengita), calcio en dos de sus formas (fosfato tricálcico o fosfato ortocálcico) y aluminio (variscita) (Useche et al., 2004). No obstante, Cabrera (2000) concluyó que el aporte que realizan algunos microorganismos al stock de fósforo del suelo es bajo, y las fuentes más abundantes como la materia orgánica son las menos sensibles a la actividad de las enzimas microbianas.

De igual forma, la solubilización de los compuestos minerales fosfatados puede darse por la acción sinérgica con otras poblaciones de organismos edáficos, como en el caso de las lombrices de tierra que pueden estimular la actividad de secreción de fosfatasas microbianas (Peña-Venegas y Cardona, 2010). Ramírez et al. (2001) encontraron que la inoculación de bacterias solubilizadoras de fosfatos con micorrizas arbusculares en Andisoles ha presentado efectos positivos en estos suelos, lo que se traduce en un incremento en la tasa de crecimiento de las plantas.

\section{Actividad microbiológica y distribución de los HFMA en el territorio amazónico}

La importancia y distribución geográfica de las micorrizas en ecosistemas amazónicos han sido ampliamente estudiadas (Gomes et al., 2011; Stürmer y Siqueira, 2011; Alves da Silva et al., 2012; Germera et al., 2012; López-Quintero et al., 2012; Posada, Madriñan y Rivera, 2012), especialmente en lo relacionado con: incremento de la absorción de fósforo y nitrógeno, producción de semillas, resistencia a patógenos, estabilidad edáfica y crecimiento vegetal.

Se ha encontrado que la presencia de las micorrizas favorece la absorción del fósforo en plantas que crecen sobre suelos ácidos o de baja a moderada fertilidad (Zavala, 2011). De igual forma, los hongos 
micorrízicos arbusculares pueden interactuar con otros microorganismos del suelo (en las raíces, en la rizósfera y en la masa del suelo), incrementando la fijación del nitrógeno, aunque por sí solos no sean capaces de hacerlo (Pérez, Rojas y Montes, 2011).

Dentro de los beneficios más visibles de la formación de la micorriza arbuscular, se encuentra la capacidad de los hongos para estimular en las plantas hospederas un mayor tamaño y producción de semillas, a través de la incorporación de fósforo y otros nutrientes (Montaño et al., 2009). Adicionalmente, se sabe que la producción de fitohormonas por parte del hongo mejora la estructura del suelo, favorece la resistencia a plagas y a la sequía (Jiménez, 2009).

En el suelo, el micelio de los hongos micorrízicos arbusculares participa en la formación de agregados por medio de la adhesión de partículas de glomalina, contribuyendo a darle estructura y estabilidad, reduciendo la erosión y mejorando la capacidad de retención del agua por el suelo (Ruiz, Rojas y Sieverding, 2011). Por tal razón, los efectos a nivel edáfico son claves para el mantenimiento de la diversidad vegetal y de los microorganismos del suelo, para la productividad y para la restauración de ecosistemas perturbados (Montaño et al., 2009).

Debido a la limitación de fósforo en los suelos de la Amazonia, el acceso a este nutriente por parte de las plantas es reducido y depende principalmente de las características de su sistema radicular, pues determina la eficiencia de exploración y absorción del mismo. En aquellas plantas con un sistema poco desarrollado, esta simbiosis se puede superar con la ayuda de los hongos formadores de micorrizas (PeñaVenegas et al., 2007). Por ejemplo, la efectividad de movilización de nutrientes hacia la planta se puede aumentar hasta 40 veces más con la micorriza, ya que un pelo radical puede absorber los nutrientes a $2 \mathrm{~mm}$ a la redonda y con las hifas del micelio extrarradical se logra explorar hasta 80 mm (Peña-Venegas y Cardona, 2010).

Asimismo, a estos microorganismos se les atribuye el incremento de la resistencia al estrés abiótico como sequedad y toxicidad por metales (Cardona, Arcos y Murcia, 2005), ya que estimulan el engrosamiento de la pared celular y producen un aumento en compuestos fenólicos, clorofila, arginina y citrulina (Peña-Venegas y Cardona, 2010). Se cree que a nivel genómico, hay secuencias de aminoácidos que codifican para la expresión de proteínas homólogas a las metalotioneínas, que le permite a hongos como Gigaspora rosea la captura de metales pesados (Guzmán-González y Farías-Larios, 2005).

Dentro de la diversidad de estos hongos, se ha podido determinar que las micorrizas arbusculares se encuentran ampliamente distribuidas por los trópicos. Por ejemplo, en el Amazonas se encuentran en el $80 \%$ de las plantas vasculares; en menor medida, las ectomicorrizas se hallan entre el 17 y $31 \%$ de las plantas (Peña-Venegas et al., 2006).

Según Arcos (2004), el género Glomus tiene una dominancia en la composición micorrizica del Trapecio Amazónico (cerca del 50\%), seguido de Acaulospora que se encuentra asociado a los suelos más ácidos y Gigaspora en suelos de loma y terraza. Por ejemplo, especies como Glomus glomerolatum, Glomus sp.3, Glomus sp.6, Glomus microaggregatum, Glomus manihotis, Glomus brohultii, 
Mas especificamente, Peña-Venegas et al. (2006) encontraron que en los suelos de las comunidades ribereñas del Trapecio Amazónico (San Juan de los Parentes, Nazareth, Macedonia, Mocagua, Zaragoza y San Martín de Amacayacu) hay presencia de especies de hongos formadores de micorrizas arbusculares asociados a distintas coberturas vegetales (Figura 1).

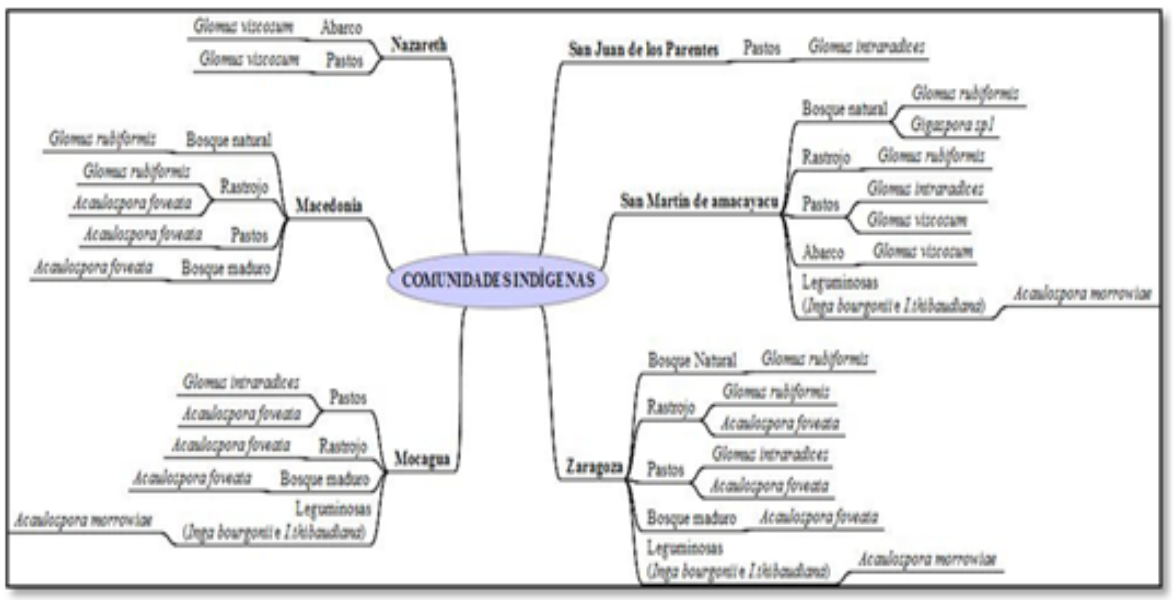

Fuente: Garzón, 2015.

Figura 1. Especies de HFMA reportadas en las comunidades indígenas ribereñas del

Trapecio Amazónico

Haga clic sobre la imagen para ampliarla

Multiples estudios (Cuenca et al., 2007; Peña-Venegas et al., 2007; Lopes, Stürmer y Siqueira, 2009; Posada et al., 2012) han analizado la relación entre la abundancia de HFMA y las características fisioquímicas del suelo, especialmente con concentraciones de fósforo. Cardona et al. (2005) determinaron la funcionalidad de los hongos formadores de micorrizas asociada con la exuberancia de la vida vegetal de la ecorregión de Bosque Húmedo Tropical. Para esto, se avaluó la abundancia de las micorrizas bajo coberturas de bosques y pastos en un paisaje fragmentado en San José del Guaviare. Encontraron que en bosques (Inga spp.) estaba la mayor cantidad de especies (con un promedio de 68,9\%), seguido de los pastos (Brachiaria decumbens) con valores de colonización que alcanzaron el $36,2 \%$.

De igual forma, determinaron que en las características fisicoquímicas del suelo como textura, acidez, saturación de aluminio, y materia orgánica, no hay diferencias significativas estadísticamente, por lo que se infiere que la variación en la abundancia de micorrizas estaría asociada a factores como la estructura vegetal de cada cobertura. La funcionalidad de la simbiosis con micorrizas arbusculares se presentó en plantas que crecen en suelos con contenidos muy bajos de fósforo (0,8-3,7 ppm), siendo el género Glomus el morfotipo más abundante en todas las rizósferas de las coberturas en las zonas de intervención evaluadas. 
Peña-Venegas et al. (2007) evaluaron la relación de las esporas de hongos glomales con las características físicas, químicas y biológicas del suelo de distintos tipos de vegetación del sur de la Amazonia. En este estudio se encontraron 18 morfotipos de esporas de HFMA diferentes: 11 especies de Glomus, 4 especies de Acaulospora, y una especie de Archaeospora, Scutellospora y Gigaspora. La presencia de fósforo soluble indicó que los suelos bajo rastrojo presentaron la mayor concentración media, valor que fue estadísticamente igual al encontrado para los suelos con cobertura boscosa, pero significativamente diferente al valor estimado para los suelos de pradera (Figura 2).

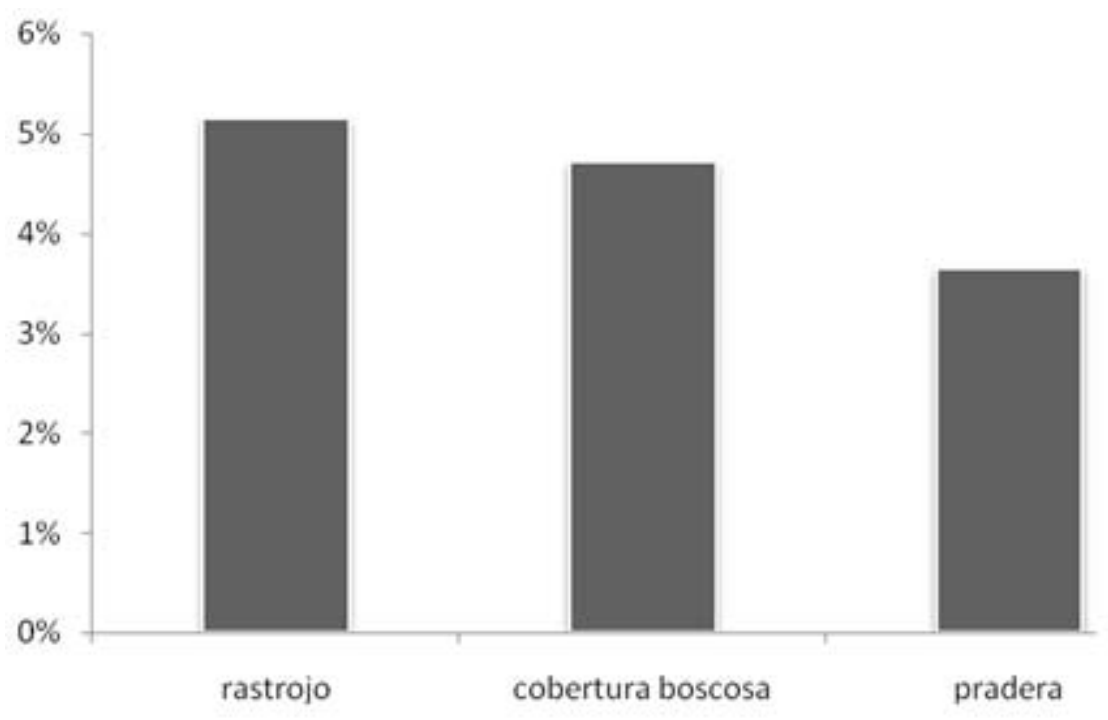

Fuente: Garzón, 2015.

Figura 2. Porcentaje de fósforo soluble en suelo de diferentes coberturas vegetales.

Lo anterior, se debe a que las plantas en estado de crecimiento segregan además mayores cantidades de exudados y enzimas que participan en la descomposición de la materia orgánica, aumentando las concentraciones de fósforo soluble en el suelo.

Para Posada et al. (2012), los factores que influyen en la distribución del inóculo de hongos micorrízicos arbusculares (micelio externo y esporas) son diversos. Entre ellos pueden mencionarse la especie vegetal predominante, el $\mathrm{pH}$, la humedad del suelo, la conductividad, el contenido de fósforo, nutrientes y de metales pesados. Evaluaron la abundancia de HFMA en el municipio de Florencia (Caquetá, Colombia), en relieves predominantes de loma y vega, con suelo usado como pastura y vegetación predominante de Brachiaria decumbens. Encontraron que los contenidos de fósforo y el $\mathrm{pH}$ en loma fueron bajos, pero la cantidad de esporas fue superior que en el relieve de vega. Asimismo, afirmaron que no parece haber una relación entre la alta variabilidad en las características microbiológicas evaluadas y las características fisicoquímicas del suelo.

Según Cuenca et al. (2007), aun cuando la tolerancia de las micorrizas a la acidez y los altos niveles de aluminio es un tema por investigar, en 
suelos ácidos tropicales tipo Ultisol estos factores no parecen afectar la funcionalidad de las micorrizas arbusculares nativas, inclusive pudiendo el hongo acumular aluminio en su micelio, vesículas y células auxiliares.

\section{Las micorrizas como herramienta para una agricultura sostenible}

En la Amazonia colombiana se ha ido adoptando la práctica de monocultivo en la población colona e indígena por la presencia de la empresa privada y de ciertas políticas estatales, lo que ha llevado a un agotamiento de los suelos y a la creación de pastizales orientados a la cría de animales (Varea et al., 1995). Asimismo, en la agricultura amazónica la aclimatación, la adaptación y la multiplicación de los cultivos en diversas condiciones agroecológicas, se han convertido en las mayores limitantes para la producción sostenible y eficiente (Noda, 2009).

Debido a lo anterior, es necesario dar paso a prácticas sustentables como la agricultura orgánica en donde los hongos formadores de micorrizas están siendo considerados como una herramienta de gran potencial (Moreira, Siqueira y Brussard, 2006). El alto costo de insumos agrícolas sumado a la creciente demanda de tecnologías menos agresivas para el ambiente, han incidido en la búsqueda de un manejo ecológico de los HFMA como una práctica que permita el desarrollo de sistemas agrícolas más eficientes (Cardona, Peña-Venegas y Arcos, 2008; Melo, Ribeiro y Sagin, 2010).

Su utilización puede darse a través del trabajo con especies nativas que, luego de determinadarse su asociación con una especie vegetal de interés, pueden ser estimuladas para potenciar el crecimiento vegetal (Klironomos, 2003). Para obtener esporas de hongos formadores de micorrizas, se pueden emplear cultivos trampa usando una combinación de suelo rizosférico y piezas de raíces, los cuales son usados para crecer plantas hospederas (Molina, Medina y Restrepo, 2006). De esta manera, se consiguen esporas más saludables y más facilmente identificables, ya que las que se obtienen en campo pueden estar parasitadas o disponibles en menor cantidad (Lopes et al., 2009).

En este sentido, existen estudios (León, 2006; Castro, 2009) que han evaluado la abundancia de HFMA asociados a plantas específicas, con el fin de determinar su grado de afinidad e incidencia. Es importante resaltar que, por las características del ecosistema amazónico, el uso sostenible del suelo para cultivos debe tener como base una aproximación de la estructura y dinámica de la vegetación natural (Corrêa et al., 2010), por lo que estas investigaciones sirven como punto de partida para determinar la potencialidad de las micorrizas en el desarrollo de proyectos de agricultura sostenible.

Peña-Venegas y Arias (2009) evaluaron el nivel de colonización de raíces con HFMA nativos en especies de leguminosas ubicadas en potreros, chagras, rastrojo y bosques. Encontraron que las muestras colectadas en potreros, rastrojo y bosques tenían un mayor porcentaje de colonización que las obtenidas en las chagras, lo que indicó que hay una mayor presencia de micorrizas en áreas naturales no intervenidas (ver Tabla 1). 
Tabla 1. Hongos formadores de micorrizas reportados por coberturas vegetales

\begin{tabular}{|c|c|c|c|}
\hline \multirow[t]{2}{*}{ HABITAT } & \multicolumn{3}{|c|}{ VARIABLES } \\
\hline & $\begin{array}{l}\text { Porcentaje de } \\
\text { colonización }\end{array}$ & $\begin{array}{c}\text { No. de } \\
\text { especies }\end{array}$ & Especies reportadas \\
\hline \multirow[t]{5}{*}{ Potrero } & $82,4 \%$ & 14 & Acaulospora foveata \\
\hline & & & Archaeospora leptoticha \\
\hline & & & Glomus sp.1 \\
\hline & & & Glomus sp.2 \\
\hline & & & Glomus sp.4 \\
\hline \multirow[t]{6}{*}{ Rastrojo } & $81,2 \%$ & 7 & Acaulospora morrowae \\
\hline & & & Acaulospora tuberculata \\
\hline & & & Glomus sp.1 \\
\hline & & & Glomus sp.2 \\
\hline & & & Glomus sp. 3 \\
\hline & & & Glomus sp.4 \\
\hline \multirow[t]{4}{*}{ Bosque } & $83,3 \%$ & 12 & Acaulospora foveata \\
\hline & & & Glomus sp.1 \\
\hline & & & Glomus sp.2 \\
\hline & & & Glomus sp.4 \\
\hline \multirow[t]{4}{*}{ Chagra } & $68,4 \%$ & 12 & Acaulospora sp.1 \\
\hline & & & Glomus sp.1 \\
\hline & & & Glomus sp. 3 \\
\hline & & & Glomus sp.4 \\
\hline
\end{tabular}

Fuente: Garzón, 2015

Sin embargo, no encontraron la misma tendencia con el número de especies de hongos micorrízicos reportados. En las leguminosas colectadas tanto en chagras como en bosques se hallaron la misma cantidad de especies, mientras que las obtenidas en el rastrojo presentaron la menor cantidad de especies.

Debido a que la dinámica de colonización de los hongos formadores de micorrizas puede variar de acuerdo con las condiciones edáficas y climáticas de una región (Barrer, 2009), es importante evaluar en el Amazonas colombiano este proceso en diferentes épocas del año para observar cómo varía la efectividad de la acción de las micorrizas, y cómo puede afectar los estudios que se vayan a realizar en una temporada específica.

Por ejemplo, Pereira y Nogeira (2006) evaluaron en Manaos, Amazonia occidental de Brasil, la colonización de micorrizas arbusculares en plantas de copoazú (Theobroma grandiflorum) y pijuayo (Bactris gasipaes) en época lluviosa y seca. Estos investigadores encontraron que en el caso 
del copoazú hubo una mayor colonización de micorrizas en época lluviosa $(21,93 \%)$ que en época seca $(7,19 \%)$. Por el contrario, el pijuayo presentó una mayor colonización en época seca $(43,95 \%)$ que en la lluviosa $(13,54 \%)$.

Otra opción de uso agrícola de los HFMA es inocular a las raíces de las plantas hongos previamente seleccionados, a los cuales se les conoce su manejo por prácticas agronómicas empleadas con anterioridad (Alarcón y Ferrera-Cerrato, 2000). No obstante, puede darse una competencia con hongos micorrízicos nativos que puede alterar su actividad microbiológica y llegar a reducir su eficiencia (Barrer, 2009).

Autores como Chaguezá (2011), Rodríguez-Morelos et al. (2011) y Ruiz et al. (2011) hablan de la importancia de la selección y manejo de los inóculos desde la fase de vivero, para que las plántulas in vitro sean lo suficientemente fuertes y posteriormente se pueda aumentar la supervivencia, calidad y crecimiento de las plantas en campo. Esta efectividad de inoculación, puede conocerse a través de la cuantificación del porcentaje de colonización del hongo en la raíz y por la determinación de las características morfológicas del mismo (Habte y Osorio, 2001).

En la zona amazónica del Guaviare, Salamanca (2003) evaluó en fase de invernadero la efectividad de la inoculación de especies de hongos micorrízicos nativas e introducidas (Acaulospora longula, Acaulospora spinosa y Glomus clarum) en las especies frutales de arazá (Eugenia stipitata), borojó (Borojoa sorbilis), chontaduro (Bactris gasipaes) y la pastura (Brachiaria decumbens). Encontró una alta colonización de las raíces en todas las especies vegetales, siendo el pasto el más alto con un $74,8 \%$, seguido del chontaduro (67\%), el borojó $(60,1 \%)$ y el arazá $(53,8 \%)$.

Los anteriores resultados mostraron que las las gramíneas como Brachiaria decumbens son micotróficas dependientes, lo que las hace más óptimas para cultivo en suelos ácidos y de baja fertilidad. Adicionalmente, se observó una alta asociación con HFMA del género Glomus (55\%), y en menor proporción con los géneros Acaulospora (27\%), Entrophospora (9\%) y Scutellospora (9\%).

Sumado a lo anterior, Cuenca et al. (2007) realizaron la inoculación de especies de HMA (Glomus manihotis, Scutellospora fulgida, Entrophospora colombiana, muy adaptadas a suelos ácidos) en plantas de la especie $V$. luteola para evaluar su cambio en la productividad. Reportaron un aumento de un $4000 \%$ del peso seco de la planta y un incremento del 315\% en la producción de biomasa. Asimismo, se dilucidó que existe una compatibilidad funcional entre micorrizas, el suelo y las plantas, por lo cual hay combinaciones mejores que otras.

Para Quintero y Pérez (2006), un verdadero uso sostenible del suelo para cultivos en Colombia debe enfocarse en el uso de las micorrizas arbusculares articuladas con el conocimiento sobre las unidades de manejo ambiental, acordes con: la tasa de extracción, la capacidad de uso, el acervo cultural de las comunidades locales y la capacidad de autorrecuperación de los ecosistemas. De esta manera, es posible desarrollar proyectos de sistemas productivos sostenibles que se adecuen a las características socioculturales y las condiciones particulares de los ecosistemas amazónicos (Vieco, 2011). 
De igual forma, las poblaciones campesinas e indígenas pueden emplear tecnologías de producción que involucren el aprovechamiento del recurso por medio del uso potencial de las micorrizas nativas como bioinsumos. Esto le permite a las comunidades mantener las condiciones naturales propias de los ecosistemas, así como mejorar la calidad nutricional de pasturas y especies de importancia alimenticia de la región (Monroy, 2004). Más específicamente, Acosta, García y Mendoza (2008) señalan la necesidad de plantear estrategias para sistemas productivos sostenibles, en las que se fusione la economía de mercado y manejo ambiental convencional con la economía y manejo ambiental indígenas, en lo que el autor denomina un "diálogo intercultural".

Arcos (2004) realizó un estudio dentro las comunidades indígenas del Trapecio Amazónico para determinar la abundancia de las micorrizas en plantas de importancia alimenticia para la población como yuca dulce y yuca brava (Manihot esculenta), maíz (Zea mays), piña (Ananas sp.) y plátano (Musa sapientum). Las variedades de yuca evaluadas siempre reportaron colonización de micorrizas entre 30 y $52 \%$ y se encontraron esporas de los géneros Glomus, Gigaspora y Acaulospora, siendo el Glomus el de mayor frecuencia y distribución.

Asimismo, señaló que los suelos de los agroecosistemas en el Trapecio Amazónico colombiano son altamente dependientes de la participación de la microbiota del suelo en procesos de descomposición, mineralización, solubilización y fijación simbiótica de nutrientes. Las características que parecen estar asociadas a una mayor actividad micorrizal fueron las reportadas en loma y terraza y corresponden a niveles bajos de fosforó, $\mathrm{pH}$ extremadamente ácido, escasos contenidos de micronutrientes y niveles altos de materia orgánica y nutrientes. Determinó que la alta presencia de cepas nativas de micorrizas en cultivos que crecen en suelos de baja fertilidad y niveles tóxicos de aluminio, es un indicador de que la simbiosis participa benéficamente como mecanismo de nutrición vegetal.

Cardona et al. (2008) evaluaron la asociación de la planta del ají (Capsicum sp.) y los HFMA en chagras y huertas de las comunidades ribereñas al río Amazonas. Reportaron que hubo un nivel medio de micorrización con hifas, que osciló entre el 33,6 y el 41,29\%. Asimismo, se observó una correlación inversa entre el porcentaje de colonización y los contenidos de fósforo disponible en los suelos de la mayoría de las accesiones evaluadas (Figura 3 ). 


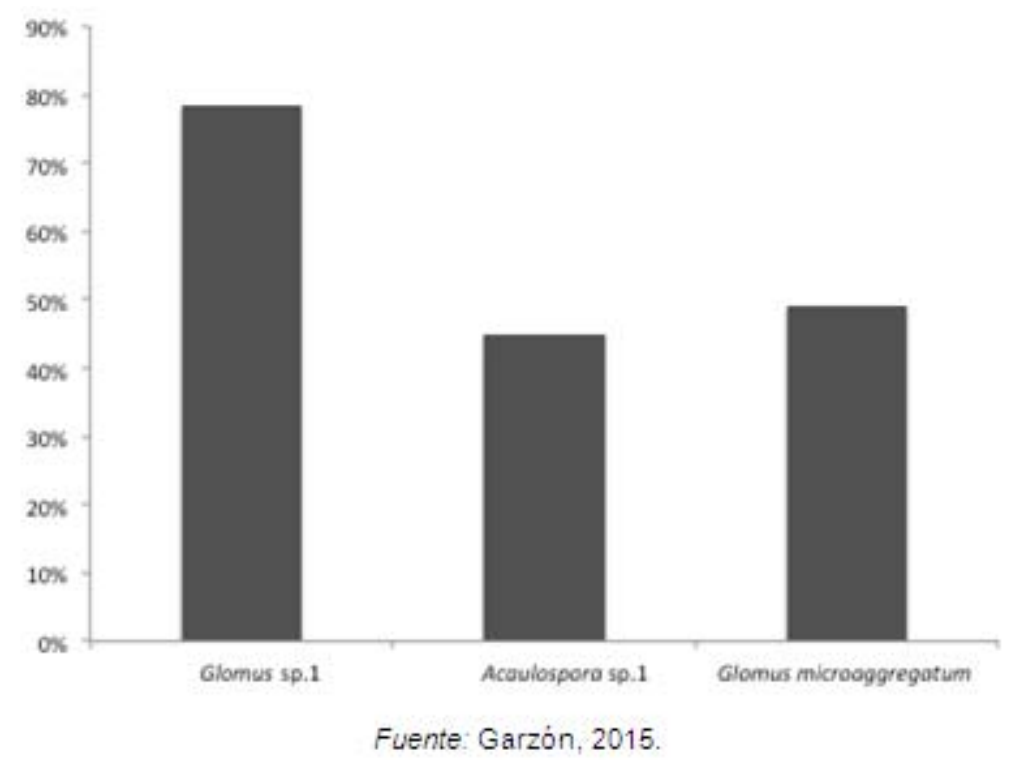

Figura 3. Porcentaje de fósforo soluble en suelo de diferentes coberturas vegetales.

Glomus sp.1 fue la especie de más amplia distribución, seguido por Acaulospora sp.1 y Glomus microaggregatum. Uno de los morfotipos, Glomus sp.5, se observó solamente en la rizósfera de una muestra de $C$. chinense proveniente del departamento de Amazonas. Sumado a esto, los investigadores encontraron una asociación entre una alta colonización micorrízica en chagras donde el cultivo predominante era la yuca, además de otros cultivos de piña y plátano. Esto se debe a que estas especies vegetales presentan una alta micotroficidad, por lo que estimulan el crecimiento y el establecimiento de cepas nativas de HFMA.

Lo anterior, se puede explicar por el hecho de que en las chagras hay un buen uso de la tierra a través de sistemas de cultivo mixto y de policultivo de diferentes hábitos de crecimiento (Cabrera, 2004; Gasché, 2006). En estos hay un gran número de especies vegetales sembradas, en donde la yuca es el producto más importante, pues se considera el "alma de la chagra"; es decir, que la supervivencia de ésta depende de que haya buenas cosechas de esta planta (Triana-Moreno, Rodríguez y García, 2006).

Las comunidades locales a través del conocimiento tradicional pueden aportar para la generación de nuevas formas de saber sobre sistemas productivos, ya que históricamente han podido transformar y mantener el entorno natural (García y Acosta, 2009). Asimismo, se puede apuntar a nuevas formas de desarrollo enfocado a actividades productivas complementarias a las prácticas económicas tradicionales, con la implementación de tecnologías orgánicas como las micorrizas (Weigel, 2001). Esto permite un diálogo de saberes que puede constituirse en un mecanismo "acelerador de oportunidades y capacidades dentro de una estrategia de desarrollo sostenible" (Gainza, Acosta y Bernal, 2008, p. 4). 


\section{CONCLUSIONES}

Existe una relación positiva entre los hongos formadores de micorrizas y otros microorganismos del suelo, especialmente con las bacterias. Esto se evidencia en el incremento del porcentaje de colonización de las raíces con estos hongos, lo que repercute en la efectividad para llevar a cabo funciones como la remoción de toxinas, la solubilización de fosfatos orgánicos e inorgánicos y la resistencia a patógenos.

De los hongos formadores de micorrizas arbusculares, el género Glomus es el que se encuentra más ampliamente distribuido en los trópicos, especialmente en el área del Trapecio Amazónico. El género Acaulospora se encuentra en menor medida y está más relacionado con suelos ácidos; mientras que la Gigaspora se encuentra principalmente en zonas de terraza y loma. Otros géneros como Archaeospora y Scutellospora tienen una baja abundancia y distribución en la zona amazónica.

Debido a los beneficios de la relación simbiotica de las plantas con los HFMA, estos microorganismos están siendo contemplados como un instrumento de gran potencial para un manejo ecológico de la agricultura amazónica, que conduzca a un uso sostenible del suelo. Su forma de empleo puede ser a través de la utilización de especies nativas de hongos específicas para una planta de interés. Sin embargo, es necesario tener en cuenta variables como las condiciones bioquímicas del suelo y la variación climática, que pueden influir en su grado de efectividad.

De igual forma, se puede trabajar cultivos in vitro en viveros donde se emplean especies de HFMA nativos o comerciales para técnicas de inoculación de raíces, con un manejo agronómico previamente conocido. A través de este método se puede establecer la compatibilidad funcional entre micorrizas, el suelo y plantas que lleve a un incremento en la producción; aunque, puede generarse una competencia negativa con otros hongos nativos.

La efectividad de inoculación de hongos formadores de micorrizas ha sido alta en especies comestibles como la yuca brava (Manihot esculenta), porotillo (V. luteola), arazá (Eugenia stipitata), borojó (Borojoa sorbilis) y chontaduro (Bactris gasipaes). Las especies más empleadas para esta técnica se encuentran dentro de los géneros de Acaulospora, Entrophospora, Scutellospora y Glomus. Este último, también se ha visto ampliamente asociado con especies vegetales de interés, por lo que se considera una especie nativa de amplia distribución y utilidad para mejorar la productividad de los cultivos en campo.

\section{REFERENCIAS}

- Acosta M., L. E., García R., O. I. y Mendoza H., D. (2008). Hacerlo amanecer: una experiencia participativa en la construcción de procesos de etnodesarrollo con pueblos indígenas en la Amazonía colombiana. Revista Colombiana Amazónica, 1, 155-173.

- Alarcón, A. y Ferrera-Cerrato, R. (2000). Biofertilizantes: importancia y utilización en la agricultura. Agricultura Técnica en México, 26(2), 191-203. 
- Alves da Silva, D. K., Rabelo, C. M., Gomes, R., Alves da Silva, G., Oehl, F. y Costa, L. (2012). Diversity of arbuscular mycorrhizal fungi in restinga and dunes areas in Brazilian Northeast. Biodiversity and Conservation, 21, 2361-2373.

- Andrews, M., Cripps, M. G. y Edwards, G. (2012). The potential of beneficial microorganisms in agricultural systems. Annals of Applied Biology, 160, 1-5.

- Arcos, A. (2004). Distribución de la asociación micorrízica en ecosistemas naturales e intervenidos. Aspectos Ambientales para el Ordenamiento Territorial del Trapecio Amazónico. Bogotá: Instituto Geográfico Agustín Codazzi.

- Barrer, S. E. (2009). El uso de hongos micorrizicos arbusculares como una alternativa para la agricultura. Facultad de Ciencias Agropecuarias, 7(1), 123-132.

- Bharadwaja, D., Lundquist, P. O. y Alströma, S. (2008). Arbuscular mycorrhizal fungal spore-associated bacteria affect mycorrhizal colonization, plant growth and potato pathogens. Soil Biology and Biochemistry, 40(10), 2494-2501.

- Cabrera, E. M. (2004). El agroecosistema "chagra" entre los indígenas en la Amazonía. Revista Luna Azul. Recuperado de http://lunazul.ucaldas.edu.co/index.php?option=com

- content\&task=view\&id=86\&ltemid=86

- Cabrera, T. (2000). Aporte al conocimiento de la microflora fúngica del suelo de la Amazonía Colombiana, con énfasis en tres grupos funcionales. Trabajo de grado, Biología. Universidad Nacional de Colombia, Bogotá.

- Cano, M. A. (2011). Interacción de microorganismos benéficos en plantas: Micorrizas, Trichoderma spp. y Pseudomonas spp. Una revisión. Revista U.D.C.A Actualidad \& Divulgación Científica, 14(2), 15-31.

- Cardona, G. (2000). Evaluación de la evolución de actinomicetos en suelos bajo tres coberturas vegetales en el Sur del Trapecio Amazónico. Tesis M.Sc. Pontificia Universidad Javeriana, Bogotá, Colombia.

- Cardona, G., Arcos, A. L. y Murcia, U. (2005). Abundancia de actinomicetes y micorrizas arbusculares en paisajes fragmentados de la Amazonia colombiana. Agronomía Colombiana, 23, 317-326.

- Cardona, G., Peña-Venegas, C. P. y Arcos, A. (2008). Ocurrencia de hongos formadores de micorriza arbuscular asociados a ají (Capsicum sp.) en la Amazonia colombiana. Agronomía Colombiana, 26(3), 459-470.

- Castro, I. (2009). Análisis de la estructura y diversidad de las comunidades de hongos formadores de micorrizas asociados a plantas de interés ecológico en ambientes mediterráneos. Tesis Doctoral. Universidad de Granada, Granada, España.

- Chaguezá, Y. (2011). Alternativas biológicas para el control de nematodos fitoparasitos en cultivo del plátano. Tesis M.Sc. Universidad Nacional de Colombia - sede Palmira, Colombia.

- Corrêa, R. S., de Oliveira, L. A., de Oliveira, A. N. y Moreira, F. W. (2010). Micorrizas arbusculares em sistemas agroflorestais na Amazônia. XXXIII Congresso brasileiro de ciência do solo, Minas Gerais, (Paper).

- Cuenca, G., Cáceres, A., Oirdobro, G., Hasmy, Z. y Urdaneta, C. (2007). Las micorrizas arbusculares como un alternativa de agricultura sustentable en areas tropicales. Interciencia, 32(1), 23-29.

- Dilantha, F. y Ru, L. (2012). Opening the Black Box: Understanding the Influence of Cropping Systems and Plant Communities on Bacterial and Fungal Population Dynamics. Ceylon Journal of Science, 41(2), 89-110.

- Fitter, A., Helgason, T. y Hodge, A. (2011). Nutritional exchanges in the arbuscular mycorrhizal symbiosis: Implications for sustainable agriculture. Fungal Biology Reviews, 25(1), 68-72. 
- Fracetto, G. G., Azevedo, L., Fracetto, F. J., Andreote, F. D., Lambais, M. R. y Pfenning, L. H. (2013). Impact of Amazon land use on the community of soil fungi. Scientia Agricola, 70(2), 59-67.

- Gainza, X., Acosta, L. E. y Bernal, H. (2008). Territorio, tecnologías del conocimiento tradicional y desarrollo. Apuntes para la Gran Amazonía continental suramericana. XI Jornadas de Economía Crítica, Universidad del País Vasco UPV/EHU, Bilbao, 27-29 marzo, (Paper).

- García, O. I. y Acosta, L. E. (2009). Resguardos indígenas y conservación del medio ambiente: Particularidades de la Amazonía colombiana. Colombia Amazónica, 4, 173-186

- Gasché, J. (2006). La horticultura indígena amazónica. Ciencias, 81, 5057.

- Germera, S., Zimmermann, A., Neill, C., Krusche, A. V. y Elsenbeer, H. (2012). Disproportionate single-species contribution to canopy-soil nutrient flux in an Amazonian rainforest. Forest Ecology and Management, 267(1), 40-49.

- Gomes, A., de Assis, H. K., da Silva, B. D., Sotão, H. M. y Baseia, L. G. (2011). Geastrum species from the Amazon Forest, Brazil. Mycotaxon, 118, 383-392.

- Guerra, B. E. (2008). Micorriza arbuscular. Recurso microbiológico en la agricultura sostenible. Tecnología en Marcha, 21(1), 191-201.

- Guzmán-González, S. y Farías-Larios, J. (2005). Biología y regulación molecular de la micorriza arbuscular. Avances en Investigación Agropecuaria, 9(2), 17-31.

- Habte, M. y Osorio, N. (2001). Arbuscular mycorrhizas: producing and applying arbuscular mycorrhizal inoculum. Honolulu, HI: University of Hawaii.

- Jesus, E., Marsh, T., Tiedje, J. M. y Moreira, F. (2009). Changes in land use alter the structure of bacterial communities in Western Amazon soils. The ISME Journal, 3, 1004-1011.

- Jiménez, V. (2009). Efecto de diferentes inoculos comerciales de hongo micorrizico arbusculares sobre "Cedrela mantana" en etapa de vivero. Trabajo de grado, Ingeniera Forestal. Universidad Distrital Fanscisco José de Caldas, Bogotá.

- King, A. (2011). Nutrient Losses in Agriculture: the Role of Biochar and Fungal. The Journal of Undergraduate Research, 11, 16-22.

- Klironomos, J. (2003). Variation in plant response to native and exotic arbucular mycorrhizal fungi. Ecology, 84(9), 2292-2301.

- León, D. (2006). Evaluación y caracterización de micorrizas arbusculares asociadas a yuca (Manihot esculenta sp.) en dos regiones de la Amazonía colombiana. Trabajo de grado, Microbiología Agrícola y Veterinaria. Universidad Pontificia Javeriana, Bogotá, Colombia.

- Lopes, P., Stürmer, S. L. y Siqueira, J. O. (2009). Occurrence and diversity of arbuscular mycorrhizal fungi in trap cultures from soils under different land use systems in the Amazon, Brazil. Brazilian Journal of Microbiology, 40(1), 111-121.

- López-Quintero, C. A., Straatsma, G., Franco-Molano, E. y Boekhout, T. (2012). Macrofungal diversity in Colombian Amazon forests varies with regions and regimes of disturbance. Biodiversity Conservation, 21(9), 2221-2243.

- Martínez, L. B. y Pugnaire, F. I. (2009). Interacciones entre las comunidades de hongos formadores de micorrizas arbusculares y de plantas. Algunos ejemplos en los ecosistemas semiáridos. Ecosistemas, 18(2), 44-54.

- Melo, E., Ribeiro, E. M. y Sagin, O. J. (2010). Comunidades de fungos micorrízicos arbusculares associados ao amendoim forrageiro em 
pastagens consorciadas no Estado do Acre, Brasil. Acta Amazónica, 40(1), 13-22.

- Molina, M., Medina, M. y Restrepo, F. (2006). Evaluación de sustratos y cultivos trampa bajo condiciones controladas para la obtención de hongos micorrízogenos de Aliso (Alnus acuminata H.B.K.). Livestock Research for Rural Development, 18(2), 1-12.

- Monroy, H. J. (2004). Caracterización de hongos formadores de micorrizas arbusculares (HMA) nativas en seis coberturas de cítricos en el Piedemonte del Meta. Trabajo de grado, Ingeniería Agrónoma. Universidad Nacional de Colombia, Bogotá.

- Montaño, N. M., Camargo-Ricalde, S. L., García-Sánchez, R. y Monroy, A. (2009). Micorrizas arbusculares en ecosistemas áridos y semiáridos. México, D.F.: Instituto Nacional de Ecología-SEMARNAT, Mundi-Prensa SA de CV, UAM-Iztapalapa, FES Zaragoza, UNAM.

- Moreira, F. M., Siqueira, J. O. y Brussard, L. (2006). Soil organism in tropical eecosystems: a key role for Brazil in the global quest for the conservation and the sustainable use of diversity. En Moreira, F. M., Siqueira, J. O. y Brussard, L. (Comps.), Soil diversity in Amazonian and other Brazilian ecosystems (pp. 1-13). Wallingford, UK: CAB International.

- Noda, Y. (2009). Las Micorrizas: Una alternativa de fertilización ecológica en los pastos. Pastos y Forrajes, 32(2), 1-10.

- Pellegrino, E., Bedini, S., Avio, L., Bonari, E, y Giovannetti, M. (2011). Field inoculation effectiveness of native and exotic arbuscular mycorrhizal fungi in a Mediterranean agricultural soil. Soil Biology and Biochemistry, 43(2), 367-376.

- Peña-Venegas, C. P. y Arias, J. C. (2009). Las leguminosas amazónicas y su importancia en la recuperación de suelos. Revista Colombia Amazónica, 2, 161-172.

- Peña-Venegas, C. P., Cardona, G., Arguelles, J. H. y Arcos, A. L. (2007). Micorrizas arbusculares del sur de la Amazonia colombiana y su relación con algunos factores fisicoquímicos y biológicos del suelo. Acta Amazónica, 37(3), 327-336.

- Peña-Venegas, C. P. y Cardona, G. (2010). Dinámica de los suelos amazónicos: Procesos de degradación y alternativas para su recuperación. Leticia: Instituto Amazónico de Investigaciones Científicas Sinchi.

- Peña-Venegas, C. P., Cardona, G., Mazorra, A, Arguelles, J. H. y Arcos, A. (2006). Micorrizas arbusculares de la Amazonia colombiana. Leticia: Instituto Colombiano de Investigaciones Científicas - Sinchi.

- Pereira, J. y Nogeira, E. J. (2006). Micorriza arbuscular em cupuaçu e pupunha cultivados em sistema agroflorestal e em monocultivo na Amazônia Central. Pesquisa Agropecuária Brasileira, 41(5), 819-825.

- Pérez, A., Rojas, J. y Montes, D. (2011). Hongos formadores de micorrizas arbusculares: una alternativa biologica para la sostenibilidad de los agroecosistemas de praderas en el Caribe colombiano. Rev. Colombiana Cienc. Anim, 3(2), 366-385.

- Phosri, C., Rodriguez, A., Sanders, I. R. y Jeffries, P. (2010). The role of mycorrhizas in more sustainable oil palm cultivation. Agriculture, Ecosystems \& Environment, 135, 187-193.

- Posada, R. H., Madriñan, S. y Rivera, E. L. (2012). Relationships between the litter colonization by saprotrophic and arbuscular mycorrhizal fungi with depth in a tropical forest. Fungal Biology, 116(7), 747-755.

- Quintero, Q. y Pérez, R. (2006). Zonificación agrícola como herramienta básica para el ordenamiento ambiental de un territorio (Caso ToluviejoSucre). Clepsidra, 2. 
- Ramírez, A., Otálvaro, D., Álvarez, C., Pérez, J. y Osorio, N. (2001). Efectos de organismos rizosféricos sobre la absorción de fosfato y el crecimiento de Leucaena en un Andisol. Suelos Ecuatoriales, 31(2), 239243.

- Rodrigues, J. L., Pellizari, V. H., Mueller, R., Baek, K., Jesus, E. da C., Paula, F. S. et al. (2012). Conversion of the Amazon rainforest to agriculture results in biotic homogenization of soil bacterial communities. Proceedings of the National Academy of Sciences, 110(3), 988-993.

- Rodríguez-Morelos, V. H., Soto-Estrada, A., Pérez-Moreno, J. y NegrerosCastillo, P. (2011). Arbuscular mycorrhizal fungi and their involvement in the production and management of neotropical forest species with emphasis on Meliaceae. Interciencia, 36(8), 564-569.

- Ruiz, P. O., Rojas, K. C. y Sieverding, E. (2011). La distribución geográfica de los hongos de micorriza arbuscular: una prioridad de investigación en la Amazonía peruana. Espacio y Desarrollo, 23, 47-63.

- Salamanca, C. R. (2003). Las micorrizas como estrategia de mejoramiento nutricional de pasturas y especies frutales en el Guaviare. Guaviare: Convenio Corpoica-Pronatta.

- Stürmer, S. L. y Siqueira, J. O. (2011). Species richness and spore abundance of arbuscular mycorrhizal fungi across distinct land uses in Western Brazilian Amazon. Mycorrhiza, 21, 255-267.

- Toro, D. R. (2004). La biodiversidad microbiana del suelo, un mundo por descubrir. Revista Luna Azul. Recuperado de http://lunazul.ucaldas.edu.co/index.php?option=com content\&task=vie w\&id=89\&ltemid $=89$

- Triana-Moreno, L. A., Rodríguez, N. C. y García, J. (2006). Dinámica del sistema agroforestal de chagras como eje de la producción indígena en el Trapecio Amazónico (Colombia). Agronomía Colombiana, 24(1), 158-169.

- Useche, Y. M., Valencia, H. y Pérez, H. (2004). Caracterización de bacterias y hongos solubilizadores de fosfato bajo tres usos de suelo en el sur del Trapecio Amazónico. Acta Biológica Colombiana, 9(2), 129-140.

- Van der Heijden, M., Bardgett, R. D. y Van Straalen, N. M. (2008). The unseen majority: soil microbes as drivers of plant diversity and productivity in terrestrial ecosystems. Ecology Letters, 11(3), 296-310.

- Varea, A. M., Vargas, M. T., Barrezueta, L. y López, J. (1995). Bosques, Árboles y Comunidades Rurales - Fase II - Documento de Trabajo: La Radio y Procesos Participativos de Desarrollo Sostenible en la Región Amazónica. Washington: FAO.

- Vieco, J. J. (2011). Desarrollo, Medio Ambiente y Cultura en la Amazonía Colombiana. III Seminario Internacional en Medio Ambiente, Biodiversidad y Desarrollo, Florencia, Caquetá, 30 de agosto al 2 de septiembre, (Paper).

- Weigel, P. (2001). O papel da ciência no futuro da Amazônia: uma questão de estratégia. Biodiversidade, pesquisa e desenvolvimento na Amazônia. Parcerias Estratégicas, 12, 62-83.

- Zavala, F. J. (2011). Rendimiento del sorgo con micorriza y fertilización química.Trabajo de grado, Ingeniería Agrónoma. Universidad Michoacana de San Nicolás de Hidalgo, Uruapan.

1. Ms en Desarrollo Sustentable y Gestión ambiental. Universidad Distrital Francisco José de Caldas. Bogotá, Colombia. lina.garzong@hotmail.com 
Para citar este artículo: Garzón, L. P. (2016). Importancia de las micorrizas arbusculares (MA) para un uso sostenible del suelo en la Amazonia colombiana. Revista Luna Azul, 42, 217-234. Recuperado de http://200.21.104.25/lunazul/index.php?option=com_content\&view=artic le\&id $=136$ 